\title{
Contraception Experience among Adolescents with Sickle Cell Disease
}

\author{
Ayana Grahama and Nancy Sokkary* \\ Department of OB/GYN, Mercer University School of Medicine and Navicent Health, USA
}

*Corresponding author: Nancy Sokkary, Department of OB/GYN, Mercer University School of Medicine and Navicent Health, 770 Pine Street, Suite 140, Macon, GA 31201, USA.

Received Date: February 07, 2020

Published Date: February 14, 2020

\section{Abstract}

Purpose: Sickle cell disease has unique impacts on females in their reproductive years. This study evaluated contraception use and knowledge among adolescent female sickle cell disease patients at a hematology clinic. Patients on hydroxyurea were predicted to report higher rates of contraception use than those not using hydroxyurea.

Methods: Female sickle cell disease patients ages 14 to 25 were surveyed regarding their contraception counseling experiences and their contraception knowledge and use.

Results: Of 12 participants, three reported having used contraception. Four reported receiving no contraception counseling. Patients on hydroxyurea did not use contraception more frequently than those not on hydroxyurea.

Conclusions: This study revealed gaps in contraception knowledge and need for better contraception counseling among female sickle cell disease patients. Further research should explore ways to increase contraception use and explore secondary effects among these patients. Collaborative care for these patients may help address all aspects of their care.

\section{Introduction}

Adolescentfemales with sicklecell disease faceuniquechallenges related to menstruation, contraception, and reproduction. Not only does pregnancy pose higher maternal morbidity and mortality risks in these patients [1], but hydroxyurea, a commonly used treatment for the disease is teratogenic [2]. Further, the frequency of vasoocclusive pain crises increases during pregnancy, which is thought to be due the associated immune modulation, hypercoagulability, and necessary suspension of hydroxyurea use [3]. Studies have indicated that women with sickle cell disease also experience greater degrees of dysmenorrhea and pains distinct from dysmenorrhea during menses $[4,5]$. Females with menstruationinduced vaso-occlusive crises often have heavier, longer menstrual periods [4]. Blood loss from menstruation can increase the risk of iron deficiency anemia, compounding the already existent sickle cell anemia.

Ideally, contraception should be explored with these patients to decrease their risk of unintended pregnancy, exposure to teratogenic agents in pregnancy, and heavy menstrual bleeding. It is recommended that patients on hydroxyurea use contraception, though the optimal contraceptive has not been defined. Due to the pain associated with menses and risks associated with unplanned pregnancies in sickle cell disease patients, the American College of Obstetricians and Gynecologists (ACOG) asserts that the benefits of combination oral contraceptives often outweigh their risk of thromboembolic events [6,7].

Adolescentfemale sickle cell disease patients should be educated on the impacts that contraception can have on overall health to allow them to make informed decisions about contraceptive use. In this small survey study, adolescent female sickle cell disease patients of a middle Georgia hematology clinic were surveyed to examine contraceptive use, depth of contraception knowledge, and experiences with contraception counseling among this population. It was hypothesized that patients on hydroxyurea would report higher rates of contraceptive use than those not on hydroxyurea. 


\section{Methods}

Prior to study initiation, the Navicent Health Institutional Review Board approved the study protocol. Patients who agreed to participate provided assent with written guardian informed consent.

\section{Subjects}

Participants were recruited during their annual visits to the Hematology Oncology Place of Excellence (HOPE) Clinic in Macon, Georgia. English-speaking female patients between the ages of 14 and 25 who were being treated for sickle cell disease met inclusion criteria. Patients with developmental delays or an inability to speak English were excluded.

\section{Procedures}

After obtaining consent, participants' names and phone numbers were collected at the time of the visit. One of two investigators then administered a confidential 24-question survey regarding patient demographics, degree of contraception counseling, and experiences with contraception use. Diagnosis and hydroxyurea use were confirmed via chart review. Patients were compensated with $\$ 10$ gift cards for participation.

\section{Analysis}

Descriptive statistics, including means, medians, and proportions, were evaluated. Utilizing Fisher's exact test due to a small sample size of 12 , findings did not approach the $p<.05$ level of statistical significance.

\section{Results}

12 adolescent female patients with sickle cell disease were recruited for and completed the study survey. All participants self-identified as African American and had a diagnosis of sickle cell disease. The age range was 14-19 years with a mean of 16.3 years $(s=1.50)$. Average age of menarche among participants was 12.7 years $(\mathrm{s}=2.47) .81 .8 \%$ reported regular monthly periods and $25 \%$ reported heavy periods, defined as more than seven days of bleeding or eight pads or tampons per day.

Three of 12 (25\%) surveyed used birth control at some point; one used medroxyprogesterone acetate ("Depo injection"), one used medroxyprogesterone acetate and a progesterone only pill, and one used a combination oral contraceptive. One patient, who experienced heavy periods and reported progesterone only pill use, reported lighter periods with less cramping after starting contraception. None of the three patients who had used birth control reported changes vaso-occlusive pain episodes.

Of the seven patients who used hydroxyurea, one had also used birth control (Figure 1). Comparatively, two of the five patients who were never on hydroxyurea had used birth control $(p=0.523$, Fisher's exact test). $50 \%$ of participants said they did not think birth control would have an effect on their pain crises, but $41.7 \%$ said they would be interested in using birth control.

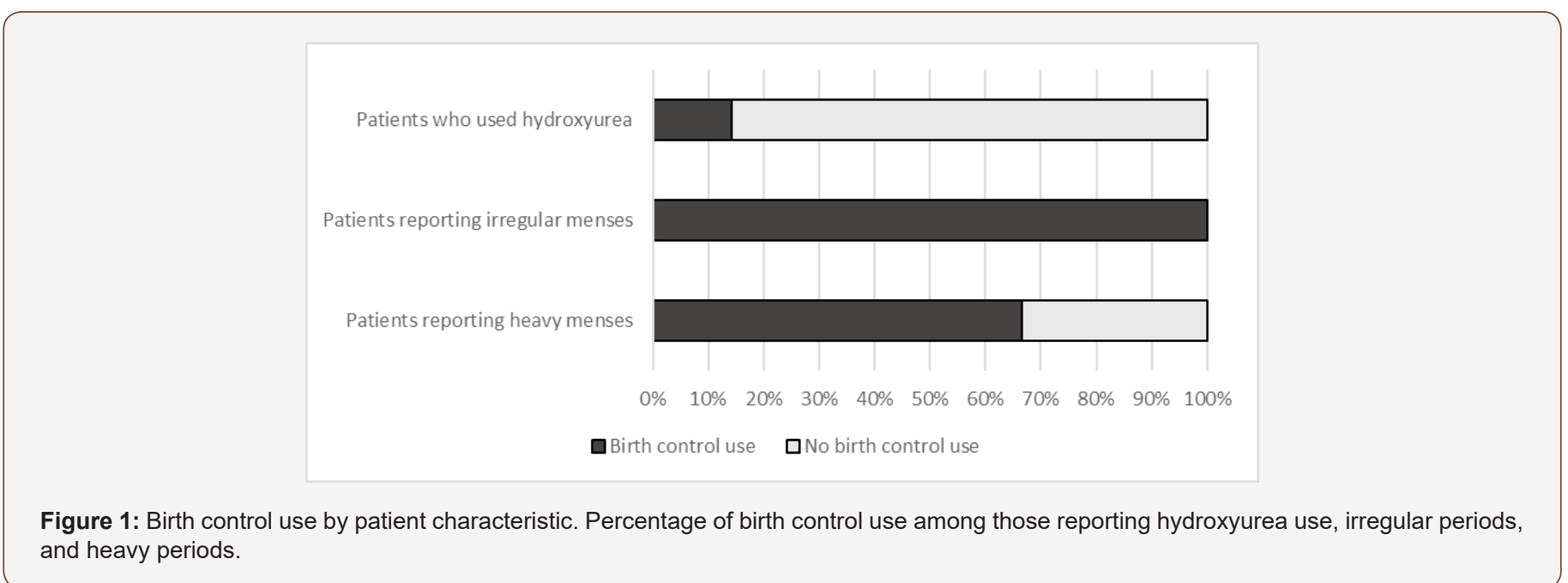

Of the 12 participants, four said no one talked to them about birth control (Figure 2). Prior to completing the survey, 10 of 12 $(83.3 \%)$ had heard of birth control pills while only one (8.3\%) had heard of the hormonal intrauterine device ("IUD", "Mirena", "Skyla", "Liletta" - Bayer, Whippany, NJ), and two were not familiar with any of the contraception types (Figure 3). To evaluate participants' general contraception knowledge, they were asked to identify the most effective form of birth control and which components of reproductive health could be improved with the use of birth control. One participant (8\%) correctly chose the intrauterine device as the most effective form of birth control, and six (50\%) knew that birth control could help with painful periods, heavy periods, and preventing pregnancy. Eight patients (67\%) said they did not believe that they knew enough about birth control.

\section{Discussion}

A large limitation on the statistical significance and generalizability of this study is its size. However, some trends can be appreciated among the sample. Contrary to predictions, in this group contraception use was higher among those not using hydroxyurea than among those who were. Additionally, many patients surveyed were not fully aware of the benefits of contraception or the range of 
options available. A third of study patients indicated that no one had and counseling may not be adequate among this patient subset. ever talked to them about birth control. Contraception education
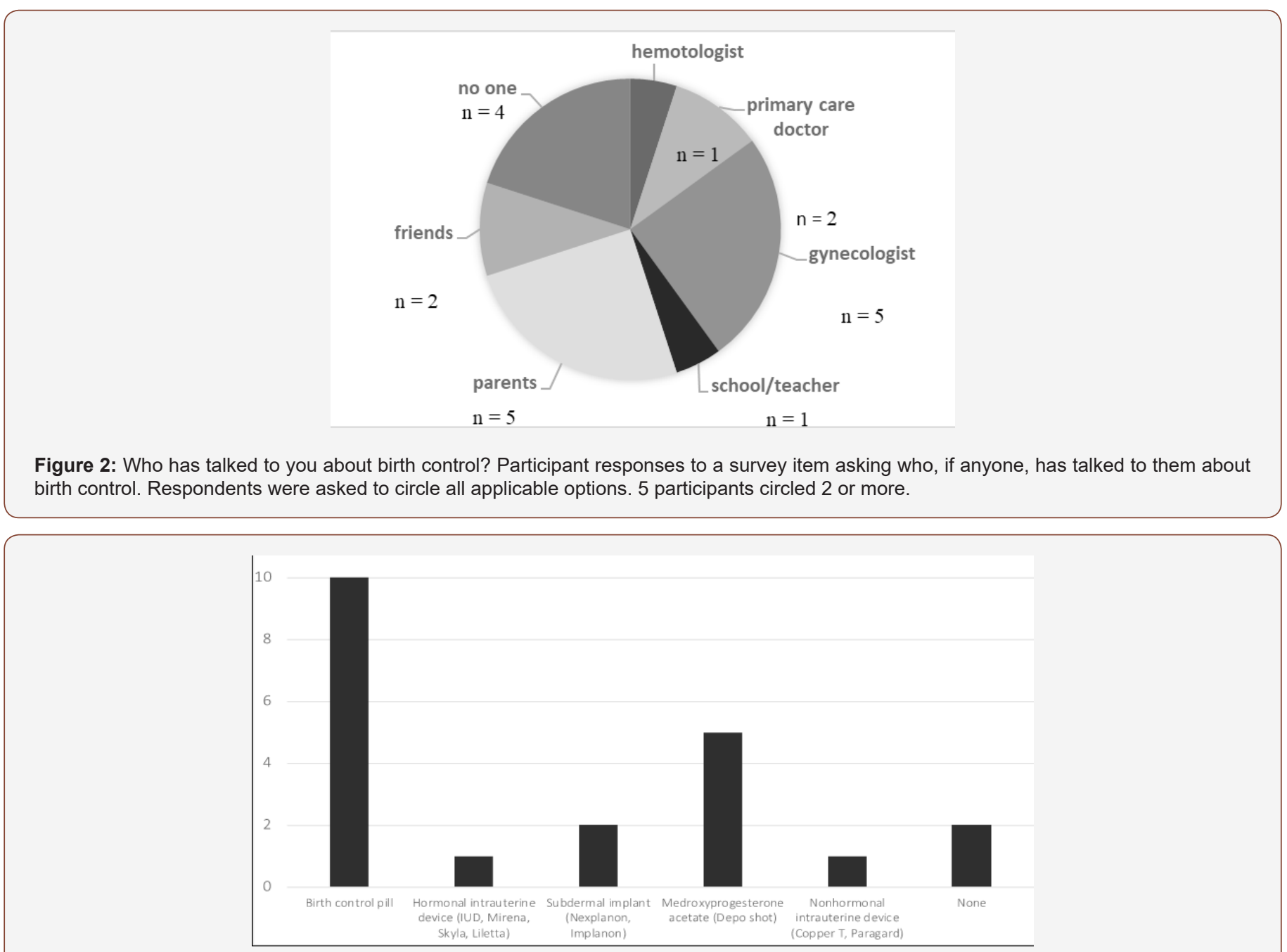

Figure 3: Number of participants familiar with each of the contraception types prior to the study survey. Nine participants were familiar with more than one contraceptive type.

To offer the most comprehensive care to sickle cell disease patients, it is recommended that providers have informative discussions about contraception and counsel these patients on their options. Improved provider familiarity with sickle cell disease, collaborative care among providers, and standardized consistent contraception counseling would be beneficial for sickle cell disease patients. Additional research is needed to better evaluate the potential effects of contraception on sickle cell disease severity and the most effective counseling methods.

\section{Acknowledgement}

This study was sponsored by a Navicent Health Research and Education Grant.

\section{Conflict of Interest}

Authors declare no conflict of interest.

\section{References}

1. Oteng-Ntim E, Meeks D, Seed PT, Webster L, Howard L, et al. (2015) Adverse maternal and perinatal outcomes in pregnant women with sickle cell disease: systematic review and meta-analysis. Blood 125: 33163325.

2. (2008) NTP-CERHR Monograph on the Potential Human Reproductive and Developmental Effects of Hydroxyurea. Triangle Park, NC: The National Toxicology Program.

3. Ahmed SG, Ibrahim UA (2017) A compendium of pathophysiologic basis of etiologic risk factors for painful vaso-occulsive crisis in sickle cell disease. Niger J Basic Clin Sci 14(2): 57-77.

4. Samuels-Reid J, Scott R (1985) Characteristics of menstruation in sickle cell disease. Fertil Steril 43(1): 139-141.

5. Sharma D, Day ME, Stimpson SJ, Rodeghier M, Ghafuri D, et al. (2019) Acute vaso-occlusive pain is temporally associated with the onset of menstruation in women with sickle cell disease. J Womens Health 28(2): 162-169.

6. ACOG Committee on Practice Bulletins-Gynecology (2006) ACOG Practice Bulletin Number 73: Use of hormonal contraception in women with coexisting medical conditions. Obstet Gynecol 107(6): 1453-1472.

7. Whitley KM (2014) Reproductive issues in sickle cell disease. Blood 124(4): 3538-3543. 